\title{
Changes in Detrended Fluctuation Indices with Aging in Healthy and Congestive Heart Failure Subjects
}

\author{
Óscar Barquero-Pérez ${ }^{1,2}$, Joaquim Marques de Sá ${ }^{1,3}$, \\ José Luis Rojo-Álvarez ${ }^{2}$, Rebeca Goya-Esteban ${ }^{1,2}$ \\ ${ }^{1}$ INEB-Biomedical Engineering Institute, Porto, Portugal \\ ${ }^{2}$ University Rey Juan Carlos, Fuenlabrada, Spain \\ ${ }^{3}$ University of Porto, Porto, Portugal
}

\begin{abstract}
Detrended Fluctuation Analysis (DFA) aims to quantify the fractal correlation properties in nonstationary time series, and it has successfully been applied in the assessment of the Heart Rate Variability (HRV) for cardiac risk stratification purposes. However the physiological meaning of DFA indices and its relation with aging have not yet been completely established. Given that a loss of complexity in the physiological regulation of elderly subjects has been reported in the literature, we hypothesized that DFA indices could be modified by aging. In this work we computed the Hurst exponent (estimated using DFA method) and DFA indices $\alpha_{1}$ and $\alpha_{2}$ to assess the HRV in healthy and Congestive Heart Failure (CHF) subjects, and we studied the dependence of these indices on aging for both healthy and CHF subjects. We found that only $\alpha_{2}$ and Hurst exponent, and only in the case of healthy subjects, have significant discrimination capability to distinguish between young and elderly groups, and also that these indices have a steady increase with aging. Therefore, we can conclude that the loss of complexity due to aging can be quantified by changes in the values of $\alpha_{2}$ and Hurst exponent.
\end{abstract}

\section{Introduction}

Healthy human heart rate has been shown to exhibit complex dynamics, which consists of both short-term and long-term temporal correlations [1]. The Detrended Fluctuation Analysis (DFA) is a method for quantifying the fractal correlation properties in non-stationary physiological time series [2], and has widely been applied for Heart Rate Variability (HRV) signals. Although a loss of complexity in the physiological regulation due to aging has been conjectured, the effect of the aging on complex heart rate dynamics in humans, and hence the physiological meaning of the changes in DFA indices and its relationship with aging, have not yet been fully elucidated [3, 4].

The purpose of this paper is to study the relationship between age and the changes in Hurst exponent and DFA indices. In order to obtain an adequate quantitative description of this relationship, we analyzed DFA indices for both healthy and Congestive Heart Failure (CHF) subjects.

The structure of the paper is as follows. In the next section we summarize the DFA algorithm for HRV analysis. In Section 3, we describe the patient data sets used in the study, as well as the scheme of the data analysis. Then, in Section 4, we present the main results obtained in terms of aging behavior and discrimination properties of the DFA indices. Finally, the conclusions and discussion are presented.

\section{DFA indices and Hurst exponent}

The DFA is a well-established method for assessing and quantifying short-term and long-term correlations in time series with non-stationarities [2]. This algorithm determines the scaling behavior of the time series based on the computation of a scaling exponent, $\alpha$, from a discrete-time process with length $N$ samples. For HRV signals, $x(n)$ is the $n$-th RR-interval between consecutive beats in an ECG. The DFA procedure consists of four steps. In the first step, the time series is integrated as follows,

$$
y(k)=\sum_{i=1}^{k}(x(i)-\bar{x})
$$

where $\bar{x}$ is the average RR-interval. In the second step, the integrated time series $y(k)$ is divided into $N_{s}$ nonoverlapping segments of equal length $s$. In the third step, 
a least squares linear regression is fitted to each segment. Coordinate $y$ of the fitted line is denoted by $y_{s}(n)$, and it represents the linear trend for each given segment. In the last step, the root-mean square fluctuation, $F(s)$, of the detrended integrated series is quantified by

$$
F(s)=\sqrt{\frac{1}{N} \sum_{k=1}^{N}\left(y(k)-y_{s}(k)\right)^{2}}
$$

This computation is repeated over all scales, i.e., segment lengths, yielding a relationship between $F(s)$, the averaged root-mean square fluctuation, and the segment length $s$. If the data $x(n)$ is self-similar, the fluctuation function $F(s)$ increases by following a power-law, that is,

$$
F(s) \propto s^{\alpha}
$$

where the fluctuation exponent $\alpha$ is related to the Hurst exponent, $H$, by $\alpha=H+1$. Therefore, it is possible to estimate $H$ using the DFA method.

Index $\alpha$ quantifies the correlation properties of a time series. For instance, a value $\alpha=1.5(H=0.5)$ indicates that the successive increments in the RR-interval time series are uncorrelated, corresponding to the Brownian motion [5]. Values of $1<\alpha<1.5(0<H<0.5)$ indicates anti-persistent behavior, i.e., the successive increments in the time series are negatively correlated. In contrast, $1.5<\alpha<2(0.5<H<1)$ indicates persistent long-term correlations, i.e., the successive increments are positively correlated. The scaling exponent $\alpha$ can be estimated as the slope of the regression line fitted to the log-log plot of the root-mean squate fluctuation function, $F(s)$ vs. $s$.

HRV signals have been found to show, at least, bi-scaling (bi-fractal) behavior. Therefore, two scaling exponents are needed in order to characterize the fractal correlations properties of the HRV signal, one for short-term, denoted by $\alpha_{1}$, and the other for long-term, denoted by $\alpha_{2}$. Practically, $\alpha_{1}$ is estimated by fitting a regression line to $\log F(s)$ vs. $\log s$, for $4 \leq s \leq 16$ (i.e less than 16 beats), whereas, $\alpha_{2}$ is similarly obtained for $16 \leq s \leq N / 4$. For very large scales, $s>N / 4, F(s)$ becomes statistically unreliable because the number of segments $N_{s}$ for the averaging procedure becomes very small [6].

\section{Patient data bases and DFA analysis}

We applied the DFA method to the RR-intervals time series obtained from 24 hour Holter recordings in healthy and CHF subjects. All data were retrieved from Physionet (http://physionet.org) [7].

The dataset for normal control group contained 72 recordings from healthy subjects from 20 to 76 years old.
Table 1. Mean \pm standard deviation for DFA indices and $H$ exponent. Significant variation $(p<0.001)$ between young and elderly groups has been highlighted.

\begin{tabular}{lccc}
\hline \hline & Young group & Elderly group & $\Delta$ Mean \\
\hline Healthy $\alpha_{1}$ & $1.245 \pm 0.162$ & $1.272 \pm 0.155$ & 0.026 \\
Healthy $\boldsymbol{\alpha}_{\mathbf{2}}$ & $\mathbf{1 . 0 0 5} \pm \mathbf{0 . 0 5 2}$ & $\mathbf{1 . 0 8 9} \pm \mathbf{0 . 0 6 5}$ & $\mathbf{0 . 0 8 4}$ \\
Healthy $\boldsymbol{H}$ & $\mathbf{0 . 0 4 2} \pm \mathbf{0 . 0 4 3}$ & $\mathbf{0 . 1 2 4} \pm \mathbf{0 . 0 4 6}$ & $\mathbf{0 . 0 8 1}$ \\
\hline \hline CHF $\alpha_{1}$ & $0.929 \pm 0.279$ & $0.977 \pm 0.237$ & 0.048 \\
CHF $\alpha_{2}$ & $1.099 \pm 0.115$ & $1.118 \pm 0.094$ & 0.019 \\
CHF $H$ & $0.113 \pm 0.061$ & $0.135 \pm 0.060$ & 0.022 \\
\hline \hline
\end{tabular}

The dataset of the CHF group contained 43 subjects from 22 to 79 years old.

All artifacts, missed detections, and ectopic beats, were excluded from the recordings, furthermore, the recordings were filtered and RR-intervals less than $200 \mathrm{~ms}$ and greater than $2000 \mathrm{~ms}$ were excluded. Also, RR-intervals were rejected if differed more than $20 \%$ from the previous and the subsequent (posterior) RR-interval [8].

We first studied the discrimination capabilities of DFA indices to distinguish between the young and the elderly subjects by splitting the dataset into two groups: Young group, from 20 to 50 years, and elderly group, from 51 to 80 years. The Student's t-test was used to check for statistically significant differences. The aim of this analysis was to elucidate whether the change in the indices between young and elderly subjects is significant or not, i.e., to elucidate whether the loss of complexity due to aging is reflected as significant variation of the fractal correlation properties.

Additionally, we studied the dependence of these indices on age for each healthy and CHF subject, by using linear regression of the indices vs. age, and then obtaining the slope (variation per year index) and its determination coefficient. The aim of this second analysis was to quantify the change of the fractal correlation properties of the HRV, i.e., to determine how evolves the change in the indices with aging.

\section{4. $\quad$ Results}

Mean values and standard deviations of DFA indices $\left(\alpha_{1}\right.$ and $\alpha_{2}$ ) and $H$ exponent for young and elderly subjects are summarized in Table 1.

The differences between young and elderly groups were significant only for $H$ exponent and $\alpha_{2}$, and only in the case of healthy subjects. None of the indices had significant differences in CHF. Though $\alpha_{1}$ was found in the literature [9] and in our results to be the best index when discriminating between healthy and pathological subjects, it showed no significant variation between young and elderly, either in healthy or in CHF subjects. This result suggests that the loss of complexity with aging is mostly due 


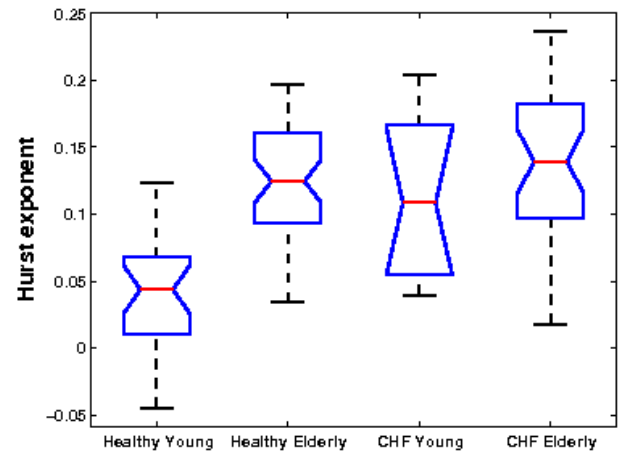

(a)

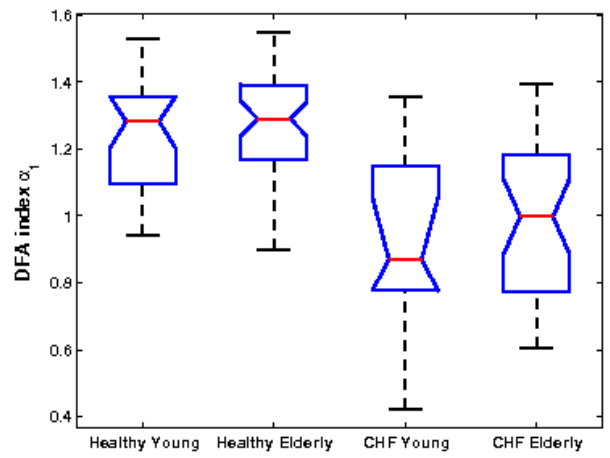

(b)

Figure 1. Notched boxplot showing the distribution of $H$ exponent (a), and DFA index $\alpha_{1}(b)$ for young and elderly groups, for both healthy and CHF subjects. $H$ exponent shows a significant difference between young and elderly for healthy subjects, that it is not shown by $\alpha_{1}$. Instead, $\alpha_{1}$ shows statistically significant differences between healthy and CHF subjects.

to the breakdown on the long-term correlations, rather than to the short-term correlations.

Figure 1 shows the notched boxplots for $H$ exponent (a) and for DFA index $\alpha_{1}$ (b). The distribution of $H$ exponent was very similar for healthy elderly group and CHF young and elderly groups, whereas for healthy young subjects the values were significantly lower. This indicates that the loss of complexity due to CHF and aging has similar effects in the change of the $H$ exponent value. DFA index $\alpha_{1}$ provided the best discrimination capability between healthy and $\mathrm{CHF}$ subjects. However, the distributions of young and elderly groups, for both healthy and CHF subjects, were very similar, that is, there were no significant differences between young and elderly. This suggests that the loss of complexity due to CHF has the effect of a breakdown on the fractal correlation characterized by $\alpha_{1}$, which affects young and elderly groups in a similar way, whereas the loss of complexity due to aging quantified by $\alpha_{1}$ provides no statistically significant differences, that is, aging seems to preserve the fractal correlation properties quantified by $\alpha_{1}$ for healthy subjects.

The variation vs. year index and the determination coefficient of DFA indices $\left(\alpha_{1}\right.$ and $\left.\alpha_{2}\right)$ and $H$ exponent in terms of their dependence on age are summarized in Table 2. $H$ exponent and $\alpha_{2}$ indices were the only ones that reveal a steady increase (linear relationship) with aging, and again only for the case of healthy subjects. DFA index $\alpha_{1}$ shows a constant value with aging, ( $\alpha_{1}$ increase $\approx 0$ units/year), wich suggests that the fractal correlation properties quantified by this index are preserved with aging, confirming the previous analysis (Figure 1(b)). All the indices have a variation for CHF subjects that can not be explained by aging in a linear way. Figure 2 shows the dependence of the estimate $H$ exponent (a), and DFA index
Table 2. Variation/year index and determination coefficient for DFA indices and $H$ exponent for both healthy and CHF subjects. Significance $(p<0.001)$ has been highlighted.

\begin{tabular}{lcc}
\hline \hline & Variation/year Indx. & Determination coef. \\
\hline Healthy $\alpha_{1}$ & $9.5690 e-004$ & 0.010 \\
Healthy $\boldsymbol{\alpha}_{\mathbf{2}}$ & $\mathbf{0 . 0 0 2 3}$ & $\mathbf{0 . 2 6 4}$ \\
Healthy $\boldsymbol{H}$ & $\mathbf{0 . 0 0 2 3}$ & $\mathbf{0 . 3 9 8}$ \\
\hline \hline CHF $\alpha_{1}$ & -0.0011 & 0.003 \\
CHF $\alpha_{2}$ & 1.017 & 0.041 \\
CHF $H$ & 1.071 & 0.041 \\
\hline \hline
\end{tabular}

$\alpha_{1}$ (b), on age for healthy subjects.

\section{Discussion and conclusions}

We used DFA indices $\alpha_{1}$ and $\alpha_{2}$, together with $H$ exponent, in order to study the dependence of the changes in these indices on aging, for both healthy and CHF subjects. First, we studied the differences between young and elderly subjects. We found that $\alpha_{2}$ and $H$ exponent provided statistically significant differences between young and elderly group, but only in the case of healthy subjects, whereas $\alpha_{1}$ provided no significant differences. This suggest that the loss of complexity due to aging causes a breakdown in the fractal properties characterized by $\alpha_{2}$ and $H$ exponent, i.e long-term correlations, whereas it preserves the fractal properties characterized by $\alpha_{1}$, i.e. shortterm correlations. Finally, we studied how the change of these indices evolves with age. We found that $\alpha_{2}$ and $H$ exponent exhibit a steady increase with age, whereas $\alpha_{1}$ exhibits a constant behavior, and only in the case of healthy subjects. We can conclude that the fractal correlation prop- 


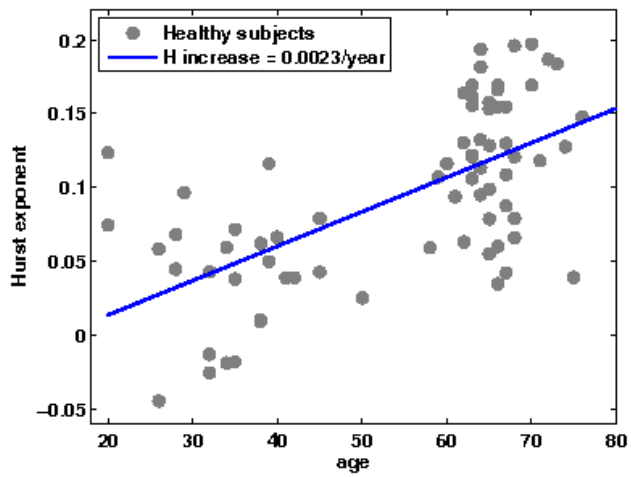

(a)

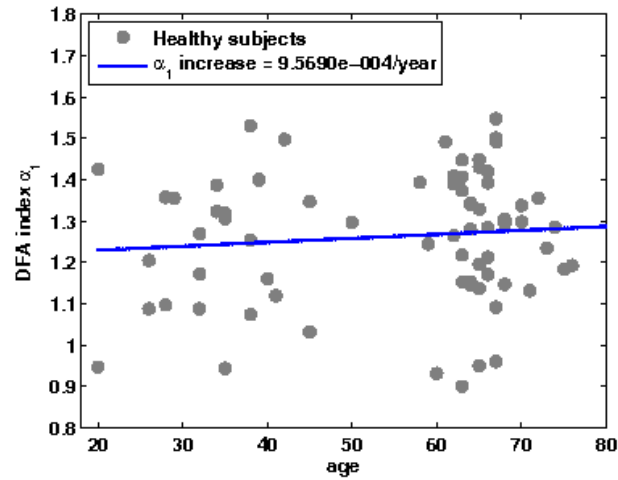

(b)

Figure 2. Dependence of estimated scaling exponents $H(a)$, and $\alpha_{1}(b)$, on age for healthy subjects. $H$ exponent shows a steady increase, whereas $\alpha_{1}$ shows a constant behavior.

erties in healthy subjects characterized by $\alpha_{1}$ are preserved with aging, whereas those characterized by $H$ exponent and $\alpha_{2}$ are not preserved.

Further studies with much larger healthy populations are needed in order to obtain a better and more accurate description of the changes in scaling exponents (DFA indices and $H$ exponent) due to aging, which will allow, ideally, to build an aging-curve of these indices for healthy subjects. Moreover, the aim is to determine the physiological relationships between the changes in the indices and the physiopathological causes, in order to use them not only as numerical indices, but rather in the clinical practice. For this purpose, the use of mathematical models that contain a detailed physiological description of circulatory system regulation processes should be considered, in order to improve our knowledge of the correlation between the information provided by the fractal indices and this regulation processes [10].

\section{Acknowledgements}

This work has been partly supported by Research Grant TEC-2007-68096-C02-TCM from Spanish Ministry of Science and Technology.

\section{References}

[1] Goldberger AL. Nonlinear dynamics, fractals, and chaos theory: Implications for neuroautonomic heart rate control in health and disease. In Bolis C, Licinnio J (eds.), The Autonomic Nervous System. Geneva: World Health Organization, 1999; .

[2] Peng CK, Havlin S, Stanley HE, Goldberger AL. Quantification of scaling exponents and crossover phenomena in nonstationary heartbeat time series. Chaos 1995;5:82-87.

[3] Goldberger AL, Amaral LAN, Hausdorff JM, Ivanov PC, Peng CK, Stanley HE. Fractal dynamics in physiology: Al- terations with disease and aging. Proceedings of the National Academy of Sciences 2002;99:119.

[4] Berger RD. Broken fractals: Where's the break? Journal of Cardiovascular Electrophysiology 2001;12(1):33-35.

[5] Mandelbrot BB, van Ness JW. Fractional Brownian motions, fractional noises and applications. SIAM Review 1968;10:422-437.

[6] Kantelhardt JW, Zschiegner SA, Koscielny-Bunde E, Havlin S, Bunde A, Stanley HE. Multifractal detrended fluctuations analysis of nonstationary time series. Physica A 2002;316:87-114.

[7] Goldberger AL, Amaral LAN, Glass L, Hausdorff JM, Ivanov PC, Mark RG, Mietus JE, Moody GB, Peng CK, Stanley HE. PhysioBank, PhysioToolkit, and PhysioNet: Components of a new research resource for complex physiologic signals. Circulation 2000 (June 13);101(23):e215e220.

[8] Malik M, Cripps T, Farrell T, Camm A. Prognostic value of heart rate variability after myocardial infarction. a comparison of different data-processing methods. Medical and Biological Engineering and Computing 1989;27:603-621.

[9] Huikuri HV, Mäkikallio TH, Perkiömäki J. Measurement of heart rate variability by methods based on nonlinear dynamics. Journal of Electrocardiology 2003;36:95-99.

[10] Rojo-Álvarez J, Sánchez-Sánchez A, Barquero-Prez O, Goya-Esteban R, Everss E, Mora-Jímenez I, GarcíaAlberola A. Analysis of physiological meaning of detrended fluctuation analysis in heart rate variability using a lumped parameter model. Computers in Cardiology 2007; $34: 25-28$.

Address for correspondence:

Óscar Barquero-Pérez, B-013

Universidad Rey Juan Carlos

Camino del Molino s/n, Fuenlabrada-28943, Madrid (Spain)

obarquero@gmail.com 